\title{
Developing a healthcare dataset information resource (DIR) based on Semantic Web
}

Jingyi Shi ${ }^{1}$, Mingna Zheng ${ }^{1}$, Lixia Yao ${ }^{2}$ and Yaorong Ge ${ }^{1 *}$

From Selected articles from the IEEE BIBM International Conference on Bioinformatics \& Biomedicine (BIBM) 2017: medical genomics.

Kansas City, MO, USA. 13-16 November 2017

\begin{abstract}
Background: The right dataset is essential to obtain the right insights in data science; therefore, it is important for data scientists to have a good understanding of the availability of relevant datasets as well as the content, structure, and existing analyses of these datasets. While a number of efforts are underway to integrate the large amount and variety of datasets, the lack of an information resource that focuses on specific needs of target users of datasets has existed as a problem for years. To address this gap, we have developed a Dataset Information Resource (DIR), using a user-oriented approach, which gathers relevant dataset knowledge for specific user types. In the present version, we specifically address the challenges of entry-level data scientists in learning to identify, understand, and analyze major datasets in healthcare. We emphasize that the DIR does not contain actual data from the datasets but aims to provide comprehensive knowledge about the datasets and their analyses.
\end{abstract}

Methods: The DIR leverages Semantic Web technologies and the W3C Dataset Description Profile as the standard for knowledge integration and representation. To extract tailored knowledge for target users, we have developed methods for manual extractions from dataset documentations as well as semi-automatic extractions from related publications, using natural language processing (NLP)-based approaches. A semantic query component is available for knowledge retrieval, and a parameterized question-answering functionality is provided to facilitate the ease of search.

Results: The DIR prototype is composed of four major components - dataset metadata and related knowledge, search modules, question answering for frequently-asked questions, and blogs. The current implementation includes information on 12 commonly used large and complex healthcare datasets. The initial usage evaluation based on health informatics novices indicates that the DIR is helpful and beginner-friendly.

Conclusions: We have developed a novel user-oriented DIR that provides dataset knowledge specialized for target user groups. Knowledge about datasets is effectively represented in the Semantic Web. At this initial stage, the DIR has already been able to provide sophisticated and relevant knowledge of 12 datasets to help entry health informacians learn healthcare data analysis using suitable datasets. Further development of both content and function levels is underway.

Keywords: Health informatics, Dataset information resource, Knowledge representation, Semantic web, Knowledge extraction

\footnotetext{
*Correspondence: yge@uncc.edu

${ }^{1}$ Department of Software and Information Systems, University of North Carolina at Charlotte, 9201 University City Blvd, 28223 Charlotte, NC, USA

Full list of author information is available at the end of the article
} 


\section{Background}

Healthcare data is rapidly growing in the era of big data. An increasing number of researchers are leveraging these datasets to improve the quality of patient care. However, challenges caused by a variety of purposes, designs, and techniques when health data were originally collected boost the complexity and diversity of healthcare datasets. For health data analysis, it requires significant time, energy, and fundamental knowledge to identify, understand, and choose the right datasets. The challenges for students and researchers who have little experience are even more pronounced. A number of online data resources, such as HealthData.gov [1], Data.CDC.gov [2], and Society of General Internal Medicine (SGIM) Research Dataset Compendium [3], integrate basic information for public datasets, which help new investigators choose datasets to a certain extent. However, the simple descriptions in these portals are hardly adequate for them to identify a suitable dataset to delve into. Simple search functions, such as a keywords search, provided by most of the resources cannot handle more complex and less concrete questions that typical novices have, such as finding existing analytical methods that are suitable for analyzing a particular dataset. Meanwhile, proprietary datasets, often having limited information in these portals, are even harder to understand and analyze.

Noticing these shortcomings, emerging research projects are attempting to build structured dataset information resources that address the challenge of dataset discovery and accessibility. For example, the Stanford University School of Medicine established the Center for Expanded Data Annotation and Retrieval (CEDAR) project in 2015 to facilitate researchers' standard use of metadata by developing an authoring-friendly computational ecosystem for metadata development, evaluation, use, and refinement [4]. By 2017, they had developed a CEDAR Workbench, which was an ontology-assisted tool to help scientific experiment metadata authoring [5]. Meanwhile, the University of California at San Diego is leading the development of a data discovery index system, the biomedical and healthCAre Data Discovery Index Ecosystem (bioCADDIE) [6], to index data that are stored elsewhere to facilitate data integration tasks that adopt content standards and high-level schema. A prototype biomedical data search engine, DataMed [7], under the bioCADDIE project, has included metadata extracted from multiple biomedical data repositories, such as the Cambridge Crystallographic Data Centre (CCDC) and U.S. National Center for Biotechnology Information (NCBI)'s BioProject. Similar to what PubMed (a free search engine that comprises more than 28 million citations from multiple literature databases and resources) has done for the biomedical literature, DataMed aims to make a comparable contribution for biomedical data.
However, the current attempts, focusing on integrating and searching datasets and dataset information, often lack consideration of the learning needs of specific target user populations. Particularly, there is no resource specifically designed to address the needs of health informatics students and novice researchers. Their learning curve is considerably steep when they explore datasets using existing resources. We believe the lack of a healthcare dataset information resource that brings information from various resources together to address the unique needs and questions from these learners is an important gap in health informatics development.

To bridge the gap, we have developed the Dataset Information Resource (DIR) framework, specifically aimed at helping entry-level health informatics students and researchers. For these novices, the challenges are different from established researchers. It is not the discovery of datasets that is important. Rather, the importance lies in the surveying of the landscape of existing datasets and the identification of a proper dataset from the set of common datasets for a given problem. Additionally, the understanding of the dataset and related analytical methods is critically important. The DIR framework does not contain actual data from the datasets. Instead, it is a specialized knowledge base that provides comprehensive knowledge and answers sophisticated questions about noteworthy datasets that address the needs of beginning learners. Besides common information about datasets, such as descriptions, we focus more on knowledge needed by novices, such as analytical methods that datasets can utilize. In this case, novices can quickly obtain a solid understanding through concrete cases. Moreover, we provide dataset blogs in the DIR so that users can easily start data analysis by following sample codes and instructions.

For a flexible, meaningful, and robust knowledge representation, we leveraged Semantic Web [8] technologies. Meanwhile, we incorporated the W3C Dataset Description Profile standard [9] developed by the Semantic Web Health Care and Life Sciences (HCLS) interest group to ensure that the metadata delivered are well defined and organized. The current DIR prototype focuses on 12 representative datasets in healthcare, including both public and proprietary datasets. The prototype is published and accessible via https://cci-hit.uncc.edu/dir/.

\section{Methods}

The DIR framework is based on Semantic Web technologies. Building on them, we developed methods to extract knowledge from the datasets as well as existing research articles that had analyzed these datasets. We also developed a question-answering module that answered novice questions that had been posted on the web. In the following sections, we briefly describe the Semantic Web first and then describe the system design, major 
components, knowledge representation and extraction, and dataset learning of the DIR framework.

\section{Semantic web}

The Semantic Web is an extension that adds semantics and logic to the well-known World Wide Web (WWW). In the traditional web pages, entities, such as concepts, are dispersed in the text. They are not clearly identified and their relationships are not explicitly represented. In contrast to traditional web pages, the Semantic Web enhances the regular web by coding and linking important concepts. Therefore, it makes semantics behind data understandable not only to human beings but also to machines. The Semantic Web is based on the Resource Description Framework (RDF) [10]. To link entities, RDF provides a straightforward syntax for describing resources, which is called "triple". An RDF triple contains three components-the subject, predicate, and the object, where the predicate represents the relationship between the subject and the object. To query the linked entities, a query language, SPARQL Protocol and RDF Query Language (SPARQL) [11], is designed, which is the key to reasoning. With the support of these techniques, a number of RDF-based resource frameworks have already been developed that show the power of the Semantic Web, such as DBpedia [12] and the Neuroscience Information Framework (NIF) [13].

\section{DIR framework overview}

The proposed architecture of the DIR system is shown in Fig. 1. It consists of three major components: (1) knowledge representation (requires the ability to represent metadata in a flexible, extendable, and reusable way to meet and surpass the FAIR Data Principles [14]), (2) question answering (delivers exact knowledge to novices), and (3) metadata extraction (extracts metadata tailored to novices from a large number of diverse dataset resources). With these components, the system can integrate and represent knowledge from scattered datasets, allow flexible research questions, and provide precise answers at a suitable level of comprehension.

The DIR prototype is built on top of the open-sourced Semantic MediaWiki (SMW) platform [15] for knowledge representation and question answering. SMW, which tightly couples traditional web pages with an RDF representation to capture essential knowledge, is an extension of MediaWiki (MW) [16] (see Fig. 2). Additionally, MW is well-known as the foundation of Wikipedia, whose English site contains 5,605,853 articles. Therefore, advantages of MW-such as stability facing massive content and heavy traffic-and advantages of SMW-including the embedded functionality to represent RDF triples by using properties, classes, and semantic forms-can be fully leveraged. Once the knowledge of diverse datasets is extracted, SMW provides a platform for representation and a SPARQL-like mechanism for the semantic query.

\section{Knowledge representation in DIR}

To represent dataset metadata in a standard manner that is findable, accessible, interoperable, and reusable, we adopt the W3C Dataset Description Profile [9] as the basis of a metadata description model. This profile categorizes dataset metadata in three levels: summary, version, and distribution (see Fig. 3). The summary level is the highest-level description of datasets for the most common information that is independent of specific versions, such as titles, publishers, and homepage links. The version level, as an intermediary of summary and distribution
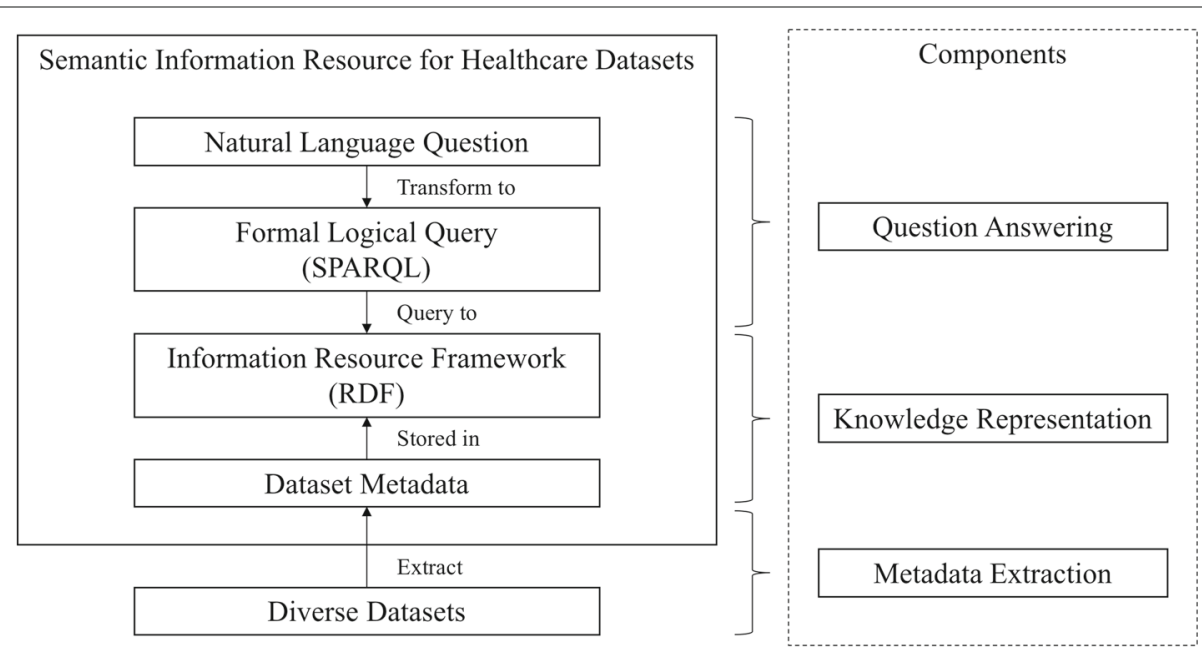

Fig. 1 Proposed architecture of DIR system 


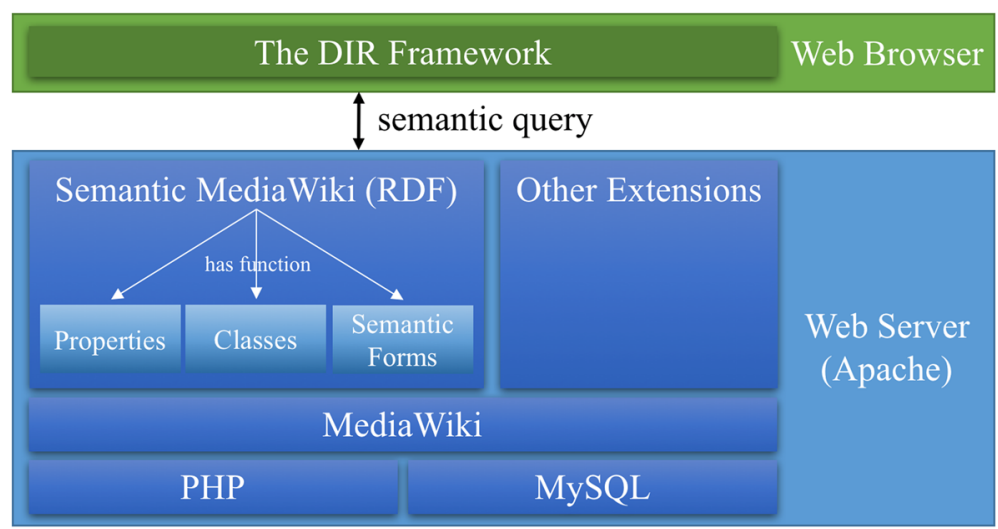

Fig. 2 Infrastructure of DIR prototype

levels, captures version-specific metadata, such as version identifiers and issue dates. The distribution level describes specific forms of a specific version. It includes the most detailed information and guidance, such as data items and links to achieve data. In the DIR prototype, each level of a dataset is a page. Since a dataset can have multiple versions and each version may have various forms, each dataset is described by at least three pages - a summary level (the entrance), at least one version level, and at least one distribution level. For each level, the W3C profile defines a set of suggested data elements, properties, and ranges. The properties that describe datasets are all selected from existing ontologies, such as the Provenance Authoring and Versioning ontology (pav) [17], Data CATalog vocabulary (dcat) [18], and the CItation Typing Ontology (cito) [19]. Since levels depend on each other, several specific properties are defined to link different level pages of a dataset, such as pav:hasCurrentVersion (links the summary level to the version level) and dcat:distribution (links the version level to the distribution level).

The DIR framework further extends the W3C Dataset Description Profile standard to incorporate properties that represent specific knowledge needed to address the

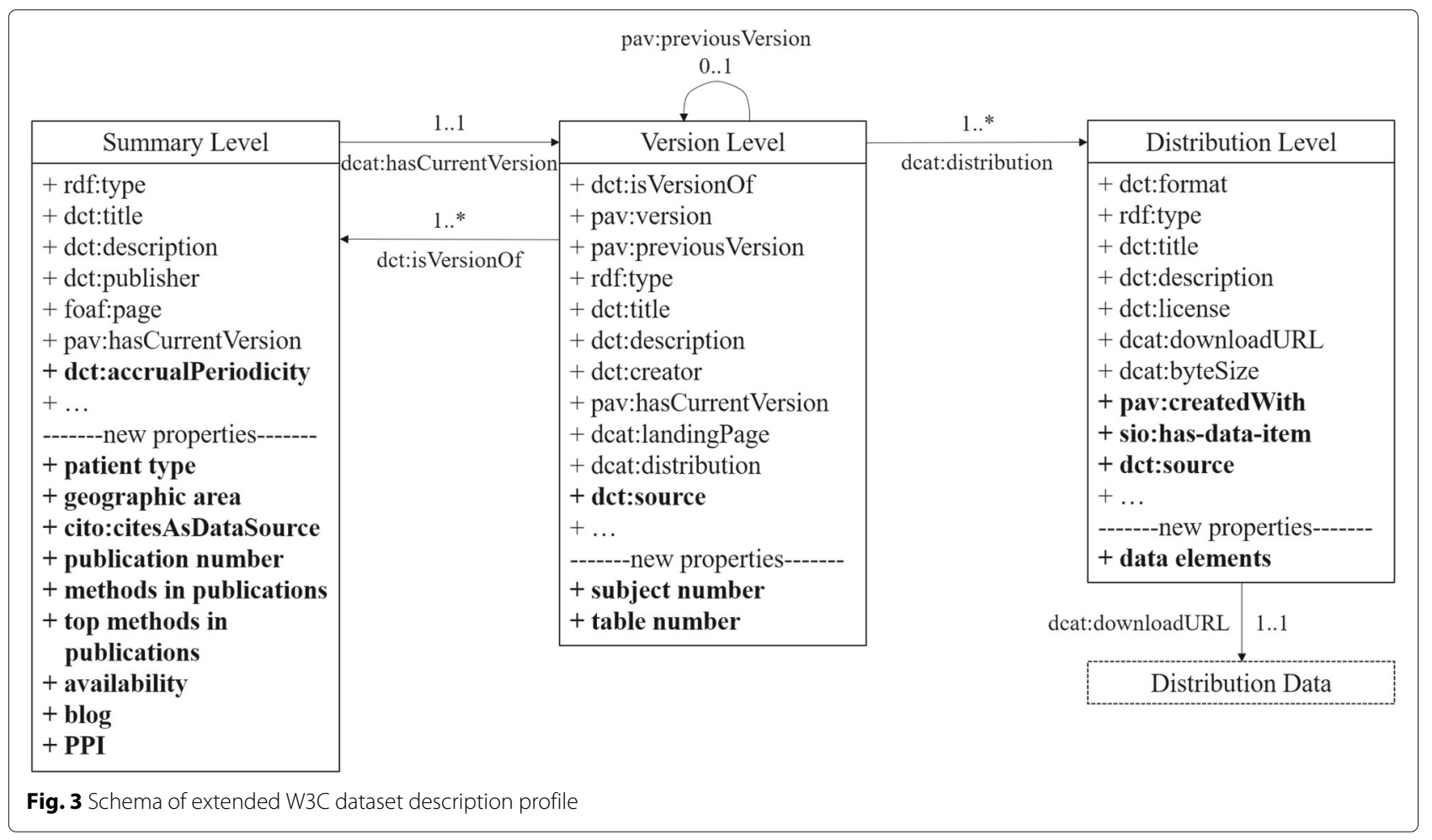


learning needs of health informatics novices. Figure 3 presents the extended properties in bold font, and Table 1 illustrates the detailed extension. As shown in Table 1, four major types of knowledge are currently extended: descriptive information, publication-related metadata, detailed data elements, and blogs. Among publicationrelated metadata, the Publication-based Popularity Index (PPI) is a special property used to compare and rank datasets [20]. Blogs of each dataset are unique and important metadata in the DIR and elaborate on concrete instructions, sample codes, and results that guide an easy start for practice. These blogs targeting novices are written by experienced dataset users, so direct support is strongly provided.

\section{Datasets in current DIR prototype}

The current implementation of the DIR includes 12 representative datasets in healthcare, of which 3 datasetsHealthcare Cost and Utilization Project (HCUP) [21], Truven Health MarketScan (MarketScan) [22], and Medical Information Mart for Intensive Care (MIMIC) [23] are retained from the previous DIR version [24]; nine others are selected from working group notes discussed by domain experts at the UNC Charlotte Health Informatics and Outcomes Research Academy [25]. The nine extended datasets are National Health and Nutrition Examination Survey (NHANES) [26], SEER-Medicare Linked Database (SEER-Medicare) [27], National Longitudinal Study of Adolescent to Adult Health (Add Health)

Table 1 Extended dataset metadata based on W3C dataset description profile

\begin{tabular}{|c|c|c|c|c|}
\hline Property & $\begin{array}{l}\text { Original value in } \\
\text { W3C profile }\end{array}$ & $\begin{array}{l}\text { Extended } \\
\text { value in DIR }\end{array}$ & Level & Description \\
\hline \multicolumn{5}{|l|}{ Descriptive information } \\
\hline dct:accrualPeriodicity & $|\mathrm{R}|$ & $\begin{array}{l}\mid \mathrm{RI} \text { or } \\
\text { xsd:string }\end{array}$ & Summary level & Dataset update frequency \\
\hline Patient type & N/A & xsd:string & Summary Level & $\begin{array}{l}\text { Patient type in a dataset } \\
\text { (e.g., ICU patients) }\end{array}$ \\
\hline Geographic area & N/A & xsd:string & Summary level & $\begin{array}{l}\text { Geographic area of a dataset } \\
\text { (e.g., city, region, and state) }\end{array}$ \\
\hline Availability & N/A & xsd:string & Summary level & $\begin{array}{l}\text { Availability of a dataset (e.g., } \\
\text { public or proprietary) }\end{array}$ \\
\hline Dct:source & $|\mathrm{R}|$ & $\begin{array}{l}\text { IRI or } \\
\text { xsd:string }\end{array}$ & $\begin{array}{l}\text { Version level and } \\
\text { distribution level }\end{array}$ & Data source provenance \\
\hline Subject number & N/A & xsd:integer & Version level & $\begin{array}{l}\text { Number of subjects (e.g., } \\
\text { number of patients) }\end{array}$ \\
\hline Table number & N/A & xsd:integer & Version level & Number of tables \\
\hline pav:createdWith & $|\mathrm{R}|$ & $\begin{array}{l}\text { IRI or } \\
\text { xsd:string }\end{array}$ & Distribution level & $\begin{array}{l}\text { Tools used to create a } \\
\text { dataset }\end{array}$ \\
\hline \multicolumn{5}{|l|}{ Publication-related metadata } \\
\hline cito:citesAsDataSource & N/A & $|\mathrm{R}|$ & Summary level & $\begin{array}{l}\text { Link to publications or a } \\
\text { collection of publications } \\
\text { using a dataset }\end{array}$ \\
\hline Publication number & N/A & xsd:integer & Summary level & $\begin{array}{l}\text { Number of publications that } \\
\text { analyze a dataset }\end{array}$ \\
\hline Methods in publications & N/A & xsd:string & Summary level & $\begin{array}{l}\text { Methods used in } \\
\text { publications to analyze a } \\
\text { dataset }\end{array}$ \\
\hline Top methods in publications & N/A & xsd:string & Summary level & $\begin{array}{l}\text { Top (usually top 10) } \\
\text { methods used in } \\
\text { publications to analyze a } \\
\text { dataset }\end{array}$ \\
\hline PPI & N/A & xsd:float & Summary level & $\begin{array}{l}\text { A publication-based } \\
\text { popularity index for dataset } \\
\text { ranking }\end{array}$ \\
\hline \multicolumn{5}{|l|}{ Detailed data elements } \\
\hline sio:has-data-item & $|\mathrm{R}|$ & $\begin{array}{l}\text { IRI or } \\
\text { xsd:string }\end{array}$ & Distribution level & $\begin{array}{l}\text { Item listing (e.g., tables and } \\
\text { entities) }\end{array}$ \\
\hline Data elements & N/A & xsd:string & Distribution level & $\begin{array}{l}\text { Data elements (e.g., } \\
\text { attributes) }\end{array}$ \\
\hline \multicolumn{5}{|l|}{ Blogs } \\
\hline Blog & N/A & $|\mathrm{R}|$ & Summary level & Links to blogs of a dataset \\
\hline
\end{tabular}


[28], Minimum Data Set (MDS) [29], Clinical Practice Research Datalink (CPRD) [30], The Health Improvement Network (THIN) [31], Premier Healthcare Database (Premier) [32], Clinformatics Data Mart (Clinformatics) [33], and Humedica NorthStar (Humedica) [34].

There are several reasons to choose these datasets. To verify the universality of DIR knowledge representation, they cover most types of healthcare datasets, including claims data (SEER-Medicare, CPRD, MarketScan, Premier, and Clinformatics), electronic medical records (MDS and THIN), hospital data (SEER-Medicare, HCUP, MIMIC, and Humedica), laboratory data (Clinformatics), surveys (NHANES and Add Health), and contextual data (Add Health).

Additionally, these datasets are all large and complex datasets in healthcare, of which three (SEER-Medicare, Add Health, and Clinformatics) even include multiple types listed in the prior paragraph. Most of them have a large number of subjects. For example, HCUP includes the largest collection of longitudinal hospital care data in the United States, and MarketScan consists of nearly 240,000,000 patients' fully integrated, de-identified, individual-level healthcare claims data. In addition to the large amount of data, the diversity of data and the complexity of the structure make novices more difficult to understand and begin to analyze the datasets. For example, MIMIC contains not only numeric and textual data stored in tabular forms, such as lab results and electronic documentation but also graphical data that are stored separately, such as bedside monitor trends and waveforms. Adopting these graphical signals requires not only a deep understanding of data themselves but also sufficient computer skills to convert them into analyzable data and adequate knowledge to decide analytical methods.

Moreover, these datasets are all widely used in healthcare data analytics. A large number of research articles have been published based on these datasets. By searching in PubMed Central (PMC) [35] —an authoritative electronic archive of free full-text biomedical and life sciences journal articles supported by U.S. National Institutes of Health's National Library of Medicine (NIH/NLM) - the most studied dataset, NHANES, was mentioned in 37,485 articles, while the least discussed dataset among them, Humedica, was mentioned in up to 22 articles. On average, each dataset contributes to more than 4000 publications in PMC.

Finally, these datasets are representative of both public and proprietary datasets. Among the 12 datasets, two of them (NHANES and MIMIC) are public for research purposes, nine of them (SEER-Medicare, HCUP, MDS, CPRD, MarketScan, THIN, Premier, Clinformatics, and Humedica) are proprietary, and one dataset (Add Health) provides both public- and contractual-use data. In novices' perspectives, complex proprietary datasets are even more challenging than public datasets because they have difficulty retrieving information elsewhere to help them build up a good understanding accurately and quickly.

In the current implementation, we manually extracted most of the metadata from dataset documentations and semi-automatically extracted metadata about analytical methods from publications. The extracted metadata was first stored in the RDF triple format in Excel spreadsheets and imported into MW, using a Python script that converts spreadsheets to MW importable XML files. To ensure the accuracy of manually extracted metadata, a team of health informatics research assistants was formed to review and correct these metadata iteratively.

\section{Extraction of analytical methods from publications}

Data analytical methods that have been successfully applied to datasets are important knowledge for data science learners. To deliver this knowledge, we developed a semi-automatic method to extract various analytical methods that had been used in published articles that analyzed the specific datasets in the DIR. For this task, we first developed an ontology of data analytical methods, Method Ontology (MethodOntology.owl [36]), which extended an existing ontology. Based on the Method Ontology, we developed a rule-based Named Entity Recognition (NER) pipeline to extract instances of analytical methods reported in selected publications.

We used PMC as the data resource and downloaded full-text articles that mentioned the 12 datasets, using the keyword identification method in [20]. In total, 48,282 PDF-format publications were obtained. The publication number of each dataset is shown in Table 2. To preprocess these publications, we developed a pre-processor, written in the Bash command and Python programming language, which included three major steps: (1) converted PDF files to plain text; (2) excluded proceedings and articles that only cited a dataset without analyzing it; and (3) selected relevant content by removing reference sections. After preprocessing, 25,201 publications remained.

The Method Ontology describes data analytical methods, which include all major machine learning, data mining, and statistical methods. This ontology extends the Data Mining Knowledge Base (DMKB.owl) of the Data Mining OPtimization Ontology (DMOP version 5.4 ), which was originally designed to support informed decision-making in the data mining (DM) process [37]. The DMKB.owl describes instances of DMOP concepts, including individual algorithms in popular data mining software, such as RapidMiner and Weka. For the method extraction purpose, the Method Ontology extended it by adding and linking new methods, which were extracted in a training set of dataset publications, and synonyms of all method instances. Figure 4 shows the structure of major 
Table 2 Publication numbers of 12 datasets

\begin{tabular}{llll}
\hline Dataset & $\begin{array}{l}\text { \# of PDF-format } \\
\text { articles in PMC }\end{array}$ & $\begin{array}{l}\text { \# for method } \\
\text { extraction after } \\
\text { preprocessing }\end{array}$ & $\begin{array}{l}\text { \# that analyzing } \\
\text { datasets }\end{array}$ \\
\hline NHANES & 37,485 & 16,213 & 10,674 \\
SEER-Medicare & 2569 & 2276 & 1627 \\
Add Health & 1881 & 1477 & 1028 \\
HCUP & 1785 & 1398 & 993 \\
MDS & 1337 & 1053 & 584 \\
CPRD & 1014 & 735 & 477 \\
MarketScan & 985 & 920 & 614 \\
THIN & 733 & 678 & 434 \\
MIMIC & 237 & 206 & 152 \\
Premier & 165 & 158 & 95 \\
Clinformatics & 69 & 65 & 49 \\
Humedica & 22 & 22 & 9 \\
Total & $\mathbf{4 8 , 2 8 2}$ & $\mathbf{2 5 , 2 0 1}$ & $\mathbf{1 6 , 7 3 6}$ \\
\hline
\end{tabular}

method classes and a few examples of extended instances in the Method Ontology.

A rule-based NER was carried out in the Clinical Language Annotation, Modeling, and Processing Toolkit (CLAMP) [38] - a Natural Language Processing (NLP) software-and was handled by a pipeline that included a sentence detector, a tokenizer, and a dictionary lookup component. The input to this pipeline included the preprocessed publications as well as a method dictionary with semantic labels generated from the Method Ontology. After all potential method entities in the publications were extracted, a post-processor was developed to refine these entities and to combine synonyms for further metadata representation. As a result, method entities were extracted and were represented on summary level pages of the datasets. Assume that a publication that analyzed a dataset mentioned at least one analytical method in the full text. In that case, more than half $(16,736$ out of 25,201$)$ of the preprocessed publications would have analyzed these datasets. According to the publications, the most frequently used methods for the 12 datasets, as well as proportions of publications that utilized the corresponding method, are shown in Table 3. Among these methods, logistic regression, mentioned in 4229 publications, was the most frequently used (see Fig. 5).

We evaluated the pre-processor and the method extraction steps separately. The results showed that the 95\% confidence interval of the pre-processor's accuracy was [92.26\%, 99.39\%], and the precision and recall of the analytical method extraction were $93.82 \%$ and $90.53 \%$, respectively.

\section{Dataset learning and question answering}

Once the dataset knowledge is extracted and represented, the direct way to query the knowledge is to write SPARQL-like queries in the semantic search mechanism provided by SMW. While this direct method is powerful, it requires an understanding of the Semantic Web and SPARQL, which is clearly burdensome to novices. Our current approach to addressing this issue is to offer a

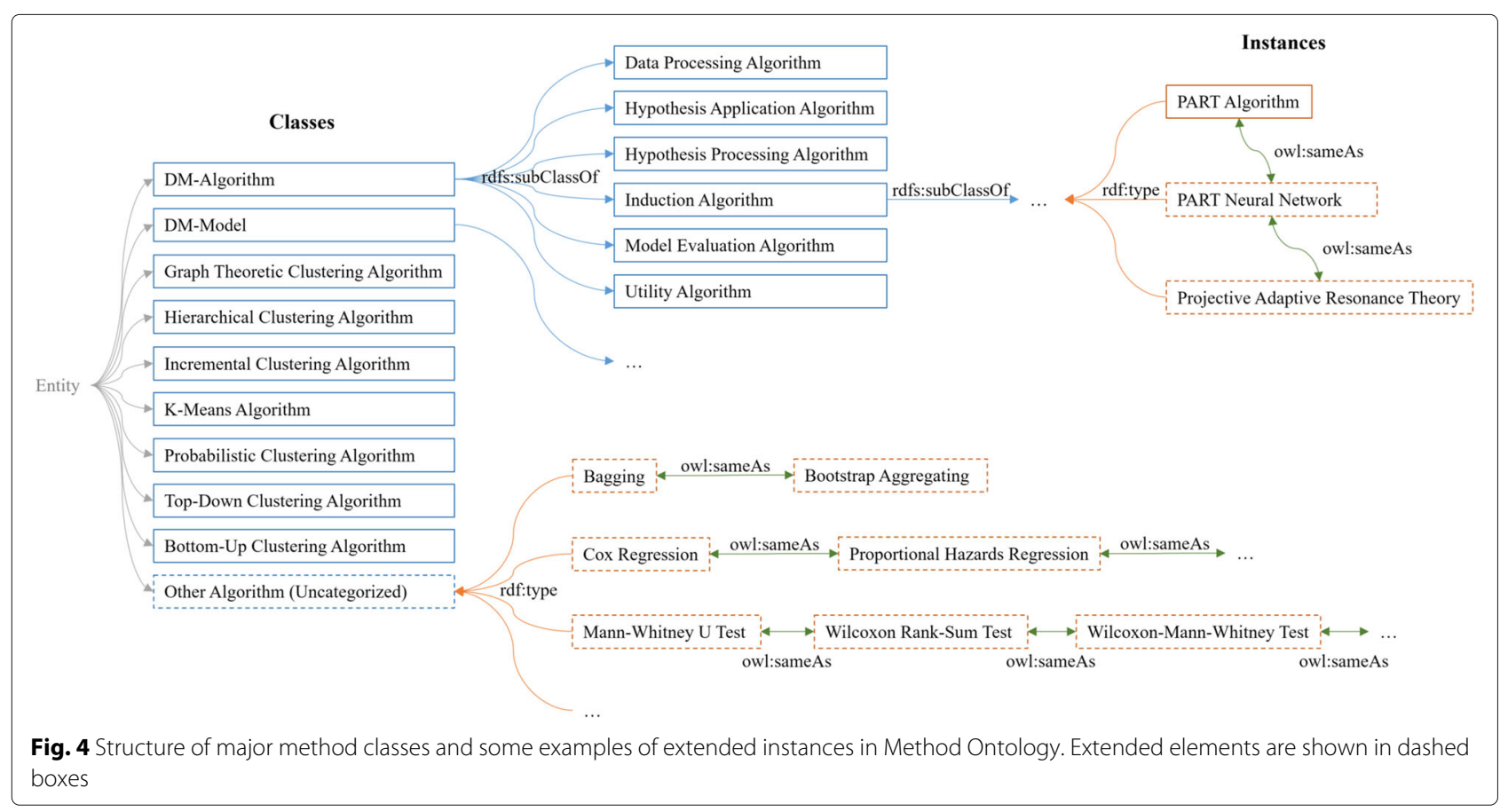


Table 3 Ten most frequently used methods to analyze each dataset

\begin{tabular}{|c|c|c|c|c|c|}
\hline Dataset & Methods & & & & \\
\hline \multirow[t]{4}{*}{ NHANES } & EM algorithm & $\begin{array}{l}\text { Neural network } \\
\text { model }\end{array}$ & $\begin{array}{l}\text { Wilcoxon } \\
\text { signed-rank test }\end{array}$ & Poisson regression & Chi-squared test \\
\hline & $29.55 \%$ & $19.63 \%$ & $16.69 \%$ & $15.02 \%$ & $14.85 \%$ \\
\hline & Kruskal-Wallis test & Logistic regression & Log-rank test & Linear regression & T-test \\
\hline & $14.32 \%$ & $12.56 \%$ & $12.17 \%$ & $10.04 \%$ & $8.51 \%$ \\
\hline \multirow[t]{4}{*}{ SEER-medicare } & Chi-squared test & Logistic regression & Cox regression & Log-rank test & Survival analysis \\
\hline & $54.52 \%$ & $50.83 \%$ & $39.64 \%$ & $17.46 \%$ & $14.87 \%$ \\
\hline & T-test & Regression model & $\begin{array}{l}\text { Kaplan-Meier } \\
\text { survival estimates }\end{array}$ & Linear regression & $\begin{array}{l}\text { Propensity score } \\
\text { matching }\end{array}$ \\
\hline & $11.12 \%$ & $10.45 \%$ & $9.34 \%$ & $8.85 \%$ & $7.01 \%$ \\
\hline \multirow[t]{4}{*}{ Add health } & Logistic regression & Chi-squared test & Linear regression & Regression model & $\begin{array}{l}\text { Principal } \\
\text { component analysis }\end{array}$ \\
\hline & $50.00 \%$ & $33.17 \%$ & $13.13 \%$ & $9.82 \%$ & $8.07 \%$ \\
\hline & ANOVA & Poisson regression & T-test & $\begin{array}{l}\text { Propensity score } \\
\text { matching }\end{array}$ & Cox regression \\
\hline & $7.49 \%$ & $5.74 \%$ & $5.06 \%$ & $3.40 \%$ & $3.40 \%$ \\
\hline \multirow[t]{4}{*}{ HCUP } & Logistic regression & Chi-squared test & Linear regression & T-test & Regression model \\
\hline & $57.91 \%$ & $48.44 \%$ & $20.24 \%$ & $18.03 \%$ & $15.61 \%$ \\
\hline & ANOVA & Poisson regression & Cox regression & $\begin{array}{l}\text { Mann-Whitney U } \\
\text { test }\end{array}$ & Bootstrap \\
\hline & $9.87 \%$ & $9.06 \%$ & $7.45 \%$ & $7.35 \%$ & $4.23 \%$ \\
\hline \multirow[t]{4}{*}{ MDS } & Logistic regression & Chi-squared test & Linear regression & Regression model & T-test \\
\hline & $42.12 \%$ & $39.73 \%$ & $17.29 \%$ & $14.90 \%$ & $13.53 \%$ \\
\hline & ANOVA & Cox regression & $\begin{array}{l}\text { Mann-Whitney U } \\
\text { test }\end{array}$ & Bootstrap & Survival analysis \\
\hline & $13.18 \%$ & $9.93 \%$ & $7.19 \%$ & $4.11 \%$ & $3.77 \%$ \\
\hline \multirow[t]{4}{*}{ CPRD } & Logistic regression & Cox regression & Chi-squared test & Poisson regression & $\begin{array}{l}\text { Propensity score } \\
\text { matching }\end{array}$ \\
\hline & $42.35 \%$ & $31.03 \%$ & $18.87 \%$ & $12.37 \%$ & $10.48 \%$ \\
\hline & Linear regression & Regression model & Survival analysis & T-test & $\begin{array}{l}\text { Kaplan-Meier } \\
\text { survival estimates }\end{array}$ \\
\hline & $9.85 \%$ & $8.60 \%$ & $6.08 \%$ & $5.66 \%$ & $4.61 \%$ \\
\hline \multirow[t]{4}{*}{ MarketScan } & Chi-squared test & Logistic regression & Cox regression & T-test & Poisson regression \\
\hline & {$[-2 p t] 47.88 \%$} & $43.32 \%$ & $19.22 \%$ & $12.87 \%$ & $12.21 \%$ \\
\hline & $\begin{array}{l}\text { Propensity score } \\
\text { matching }\end{array}$ & Linear regression & Regression model & ANOVA & Fisher's exact test \\
\hline & $10.91 \%$ & $9.93 \%$ & $9.77 \%$ & $6.68 \%$ & $5.86 \%$ \\
\hline \multirow[t]{4}{*}{ THIN } & Logistic regression & Cox regression & Chi-squared test & Poisson regression & Regression model \\
\hline & $37.33 \%$ & $26.04 \%$ & $23.27 \%$ & $12.44 \%$ & $9.91 \%$ \\
\hline & $\begin{array}{l}\text { Inverse probability } \\
\text { weighting }\end{array}$ & Linear regression & T-test & Survival analysis & $\begin{array}{l}\text { Propensity score } \\
\text { matching }\end{array}$ \\
\hline & $8.99 \%$ & $8.53 \%$ & $8.06 \%$ & $6.91 \%$ & $6.68 \%$ \\
\hline \multirow[t]{4}{*}{ MIMIC } & Logistic regression & Chi-squared test & T-test & $\begin{array}{l}\text { Mann-Whitney U } \\
\text { test }\end{array}$ & Regression model \\
\hline & $45.39 \%$ & $20.39 \%$ & $17.76 \%$ & $15.79 \%$ & $14.47 \%$ \\
\hline & $\begin{array}{l}\text { Support vector } \\
\text { machine }\end{array}$ & Linear regression & Cox regression & $\begin{array}{l}\text { Kolmogorov- } \\
\text { Smirnov } \\
\text { test }\end{array}$ & K-nearest neighbors \\
\hline & $14.47 \%$ & $11.84 \%$ & $11.18 \%$ & $9.87 \%$ & $9.21 \%$ \\
\hline \multirow[t]{4}{*}{ Premier } & Chi-squared test & K-means & Decision tree model & Logistic regression & $\begin{array}{l}\text { Propensity score } \\
\text { matching }\end{array}$ \\
\hline & $41.05 \%$ & $38.95 \%$ & $27.37 \%$ & $21.05 \%$ & $14.74 \%$ \\
\hline & Kruskal-Wallis test & $\begin{array}{l}\text { Linear discriminant } \\
\text { analysis }\end{array}$ & Regression model & Linear regression & T-test \\
\hline & $13.68 \%$ & $11.58 \%$ & $11.58 \%$ & $8.42 \%$ & $8.42 \%$ \\
\hline
\end{tabular}


Table 3 Ten most frequently used methods to analyze each dataset (Continued)

\begin{tabular}{llllll}
\hline Dataset & Methods & & & & \\
\hline Clinformatics & Linear regression & Bootstrap & Regression model & Kruskal-Wallis test & Chi-squared test \\
& $44.90 \%$ & $28.57 \%$ & $20.41 \%$ & $14.29 \%$ & $12.24 \%$ \\
& F-test & Cox regression & Logistic regression & ANOVA & Survival analysis \\
& $12.24 \%$ & $10.20 \%$ & $10.20 \%$ & $8.16 \%$ & $6.12 \%$ \\
Humedica & Chi-squared test & Logistic regression & Bootstrap & Fisher's exact test & Cox regression \\
& $33.33 \%$ & $22.22 \%$ & $22.22 \%$ & $22.22 \%$ & $11.11 \%$ \\
& T-test & Linear regression & Propensity score & Survival analysis & Ensemble learning \\
& & & matching & $11.11 \%$ & $11.11 \%$ \\
\hline
\end{tabular}

simplistic question-answering functionality by identifying the most popular questions that novices ask and providing ready-to-use queries. We created a parameterized question page for each representative question, where users can simply input words and click the Run Query button to obtain precise answers. The list of current parameterized question pages is shown in Table 4.

For example, if users are curious about which datasets can successfully utilize the Support Vector Machine, they can simply visit the "Which datasets can I apply the method to" question page, choose or type in "Support Vector Machine," and click the Run Query button to obtain "Answer: NHANES, CPRD, THIN, HCUP, MDS, MIMIC." The dataset result is in order based on the PPI recommendation. In this example, the query below has already been embedded in the question page template:

Answer:

\{\{\#ask: [ [Category:Summary Level]]

[ [Methods in publications: : $\{\{$ method $\mid\}\}\}]]$

sort=PPI

| order $=$ desc

\}\}.

As another example, if users need to investigate large datasets that have more than $1,000,000$ subjects, they can refer to the parameterized question page-"Which datasets have more than a specific number of subjects" that includes the following query:

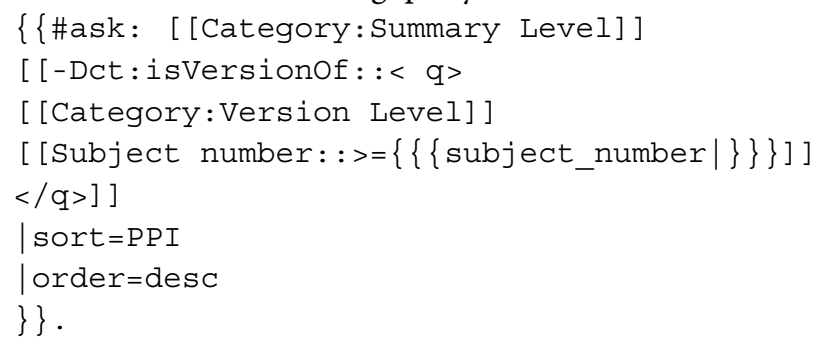

To determine the most popular questions that novices ask, we analyzed a variety of resources, including a publication that guides novices to conduct high-value dataset analysis [39], questions labeled as "dataset" on question-and-answer sites (e.g., Quora [40] and Stack Exchange [41]), and opinions from health informatics novices through interviews.

\section{Results and discussion}

A prototype of the DIR has been developed and released. It is accessible via https://cci-hit.uncc.edu/dir/. The current DIR homepage is shown in Fig. 6. Built on the foundation of the Semantic Web and the extended W3C dataset

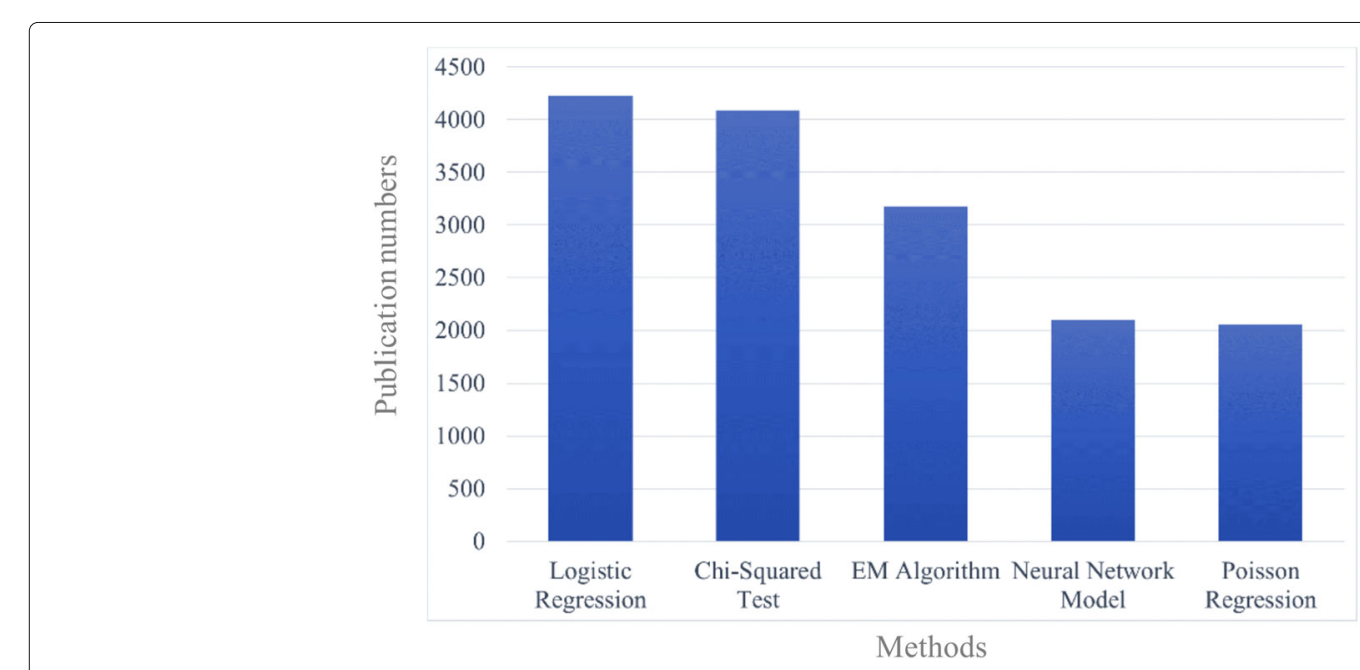

Fig. 5 The most frequently used methods in publications of 12 datasets 
Table 4 Eighteen parameterized question pages in current DIR

\begin{tabular}{ll}
\hline Data-driven questions & Which datasets include some specific \\
information/data elements? & Which datasets have more than a specific \\
& number of subjects? \\
Method-driven questions & Which datasets can I apply a specific \\
Introduction questions & method to? \\
& What does a dataset talk about? \\
& How to get a specific dataset? \\
& What are the methods that publications \\
& Used with a specific dataset? \\
& What are the publications using a specific \\
& dataset? \\
& Is a specific dataset open to the public? \\
& How many subjects are there in a specific \\
& dataset? \\
& How many tables are there in a specific \\
& dataset? \\
& What are the different tables/files in a \\
& database? \\
& What are the data elements in a specific \\
dataset? & \\
& What are the patient types that a specific \\
& dataset handles? \\
& How frequently are data updated in a \\
dataset? & How many times is a dataset cited? \\
& Who reports the data in a specific dataset? \\
& What is the geographic area of a dataset? \\
& What is the full name of a dataset? \\
& \\
\hline &
\end{tabular}

description profile, we have provided knowledge about 12 representative datasets in healthcare-NHANES, SEERMedicare, Add Health, HCUP, MDS, CPRD, MarketScan, THIN, MIMIC, Premier, Clinformatics, and Humedicaand five blogs. To facilitate novices' question answering, 18 ready-to-use questions (Table 4) have been provided. In addition, the more powerful semantic search function is available for users who are familiar with SPARQL. To ease usability, a tutorial and a support page with an issue tracker and a feedback form are also provided.

At the time of this paper's submission, the DIR prototype contained 264 pages. The average page loading time was $1.44 \mathrm{~s}$. The current approach to add a new dataset includes both manual metadata extractions (from documentations with team review-based quality check) and semi-automatic knowledge extractions (from publications using NLP technologies). To add a new dataset, the current approach takes approximately one day, in general, for manual extractions and a few minutes for semiautomatically extracting analytical methods, excluding the time to collect publications.
We have conducted a survey and collected feedback from 15 target users who were novices in healthcare data research. Of the target users, $40 \%$ had a background in health informatics, and $86.7 \%$ had a background in data analytics. We asked the subjects to compare Google, DIR, and other resources in seven use cases and also asked for general comments. The survey results indicated that 73.3\% of users, on average, preferred the DIR in these use cases. Significantly, $100 \%$ of them preferred the DIR in the case of finding datasets that included a particular data element; $93.3 \%$ preferred the DIR when they wanted to adopt a specific analytical method; and $86.7 \%$ preferred the DIR in the case of discovering large-enough datasets, such as a dataset that had more than 1,000,000 subjects. In terms of more general knowledge, users tended to rely on broader resources. For example, only $60 \%$ of users chose the DIR when they were looking for basic descriptions of a dataset or tutorials about gaining access, while others felt more comfortable on searching in Google, browsing the official website, or using both DIR and other resources simultaneously. Overall, the DIR obtained a score of $86.7 \%$ in helpfulness, $83.8 \%$ in ease of discovering datasets, $82.9 \%$ in ease of question answering, and $82.9 \%$ in the scale of meeting users' expectations about healthcare dataset information resources.

According to comments in survey responses, users highlighted the advantages of the DIR as targeted and novicefriendly. As some users commented: "It filters out the irrelevant information and is more structural"; "Beginnerfriendly. Information is exhibit[ed] clearly to the user"; and "Sample questions and semantic search are very useful for researchers to find the right dataset or information, or we can say it looks more intelligent than other search engine[s] like [G]oogle."

However, the DIR clearly has several limitations in this initial phase. (1) The current DIR prototype still relies on manual extractions in part, which is time-consuming and labor-intensive for DIR developers during dataset extending. This limitation has two possible ways to be improved. One refers to the entity linking and typing topic that is intensely discussed in Semantic Web conferences, such as the Open Knowledge Extraction Challenge (OKE) [42] at the European Semantic Web Conference (ESWC). The other way, mentioned by the CEDAR project, involves promoting an authoring-friendly ecosystem in the healthcare dataset community and encouraging researchers to contribute open metadata. (2) Currently, we do not differentiate subclasses of analytical methods, that is, the statistical methods, such as Chi-Square Test, are listed together with machine learning methods, such as Ensemble Learning. Further classification of methods based on the Method Ontology will be needed to address more detailed user questions. (3) As one user commented in the survey: "For now, finding a question is not that hard. 


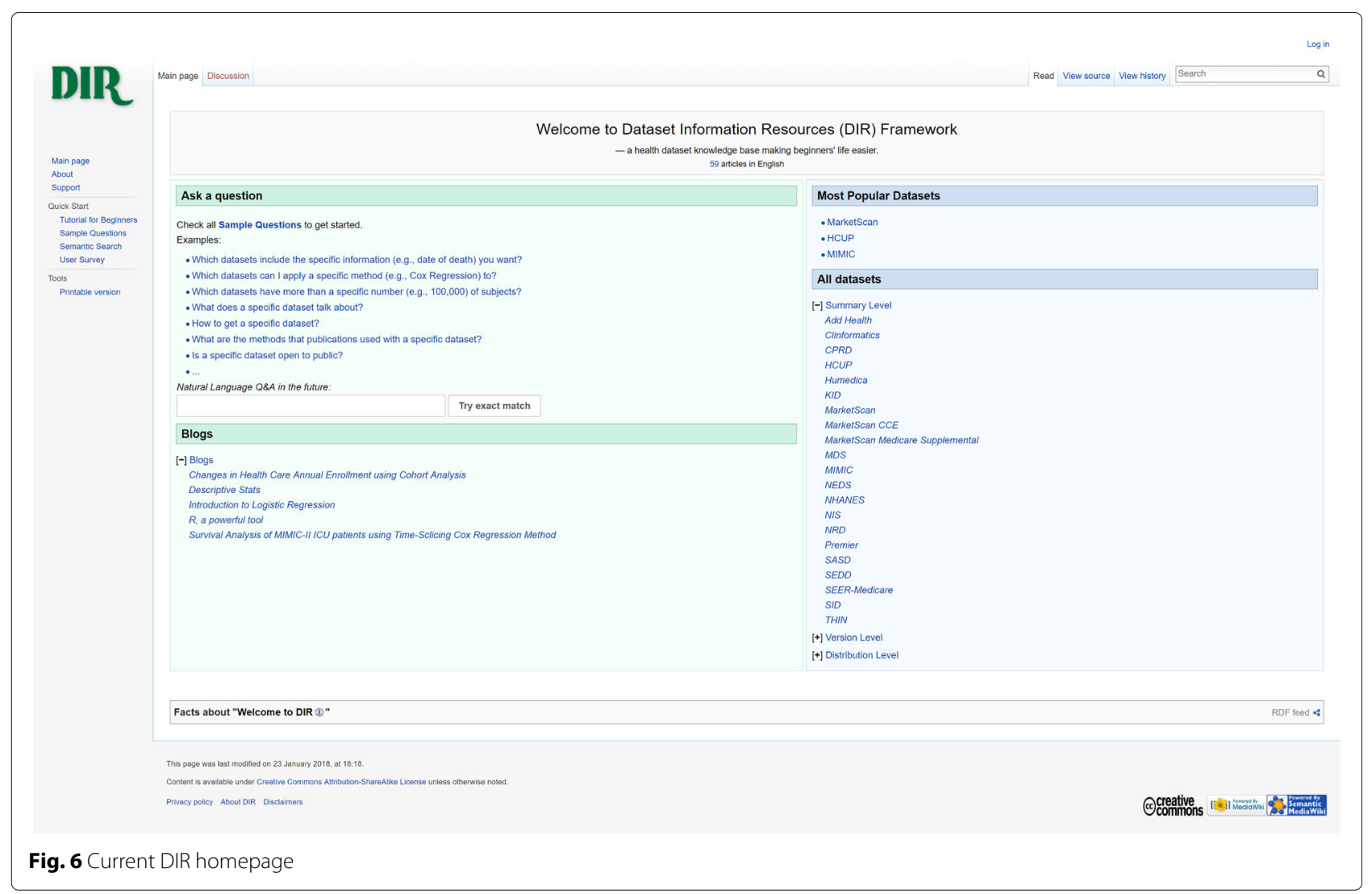

However, if the question set becomes larger, then I think it can cause a problem. Somehow you need to facilitate this part," which reveals that preparing query-embedded question-answering pages can only be a temporary solution. When the system is expanded, a real natural language question-answering functionality should be implemented. Actually, question answering is a stand-alone topic in the Semantic Web community and has been discussed over decades in conferences (e.g., the open challenge on Question Answering over Linked Data (QALD) [43] at ESWC) and publications (e.g., [44-48]). (4) As another user mentioned in the survey: "I'm not sure if researchers will trust the information on DIR." Rely on simple quality check approaches is one of the limitations. To ensure quality and to gain user trust, a systematic quality assurance method needs to be developed and reported.

\section{Conclusions}

We conclude that it is feasible to develop a DIR that provides value for entry-level health informatics students and researchers. Knowledge about datasets is effectively represented in Semantic Web technologies. At this stage, the DIR has already been able to provide comprehensive and relevant knowledge of 12 important healthcare datasets, which is expected to improve health informatics novices' ability to learn data analysis using suitable datasets.
In contrast to bioinformatics datasets, of which most data elements have already been represented in RDF at the knowledge level, the DIR will continue focusing on the healthcare datasets that are usually at a lower level granularity.

Further development is underway to improve efficiency, accuracy, and scalability. Suitable directions for expansion include two levels: content and function. The content level adds more healthcare datasets, identifies more types of knowledge for target users, and involves a systematic quality assurance method to ensure the quality of metadata. The function level includes developing a natural languagebased question-answering component, more automated methods to extract knowledge, intelligent functionalities to compare similar datasets, and collaborative features, such as discussion forums that allow users to help each other and suggest new content.

\footnotetext{
Abbreviations

Add Health: National longitudinal study of adolescent to adult health; bioCADDIE: Biomedical and healthcare data discovery index ecosystem; CCDC: Cambridge crystallographic data centre; CEDAR: Center for expanded data annotation and retrieval; cito: Cltation typing ontology; CLAMP: Clinical language annotation, modeling, and processing toolkit; Clinformatics: Clinformatics data mart; CPRD: Clinical practice research datalink; dcat: Data catalog vocabulary; DIR: Dataset information resource; DM: Data mining; DMKB: Data mining knowledge base; DMOP: Data mining optimization ontology; ESWC: European semantic web conference; FAIR: Findable, accessible, interoperable, and re-usable; HCLS: Health care and life sciences;
} 
HCUP: Healthcare cost and utilization project; Humedica: Humedica NorthStar; MarketScan: Truven health MarketScan; MDS: Minimum data set; MIMIC: Medical information mart for intensive care; MW: MediaWiki; NCBI: National center for biotechnology information; NER: Named entity recognition; NHANES: National health and nutrition examination survey; NIF: Neuroscience information framework; NIH: National institutes of health; NLM: National library of medicine; NLP: Natural language processing; OKE: Open knowledge extraction challenge; pav: Provenance authoring and versioning ontology; PMC: PubMed central; PPI: Publication-based popularity index; Premier: Premier healthcare database; QALD: Question answering over linked data; RDF: Resource description framework; SEER-Medicare: SEER-medicare linked database; SGIM: Society of general internal medicine; SMW: Semantic MediaWiki; SPARQL: SPARQL protocol and RDF query language; THIN: The health improvement network; WWW: World wide web

\section{Acknowledgements}

We would like to show our gratitude to our colleagues in the Health Informatics Lab at UNC Charlotte: Madhusudhan Vaddi, Pujitha Gangarapu, Lohitaksh Yogi, Shweta Rajaram Patil, Saugat Karki, and Jessica Yin, for their hard work in the manual metadata extraction and review; and Ishita Roychowdhury, for her important contribution in annotating analytical methods in research articles.

\section{Funding}

Publication costs were funded by faculty research support.

\section{Availability of data and materials}

Most of the data that support this study are publicly available in the DIR framework with the link https://cci-hit.uncc.edu/dir. The publications analyzed during this study are publicly available in the PMC with the link https://www. ncbi.nlm.nih.gov/pmc.

\section{About this supplement}

This article has been published as part of BMC Medical Genomics Volume 11 Supplement 5, 2018: Selected articles from the IEEE BIBM International Conference on Bioinformatics \& Biomedicine (BIBM) 2017: medical genomics. The full contents of the supplement are available online at $\mathrm{https}: / / \mathrm{bmcmedgenomics}$. biomedcentral.com/articles/supplements/volume-11-supplement-5.

\section{Authors' contributions}

$J S, M Z, L Y$, and YG contributed to the design of the research. JS developed the system, analyzed the results, and wrote the manuscript. YG supervised the research. All authors read, edited, and approved the final manuscript.

\section{Ethics approval and consent to participate}

Not applicable.

\section{Consent for publication}

Not applicable.

\section{Competing interests}

The authors declare that they have no competing interests.

\section{Author details}

${ }^{1}$ Department of Software and Information Systems, University of North Carolina at Charlotte, 9201 University City Blvd, 28223 Charlotte, NC, USA. ${ }^{2}$ Department of Health Sciences Research, Mayo Clinic, 200 First Street SW, 55905 Rochester, MN, USA.

\section{Published: 20 November 2018}

\section{References}

1. HealthData.gov. https://www.healthdata.gov/. Accessed 5 Apr 2018.

2. Data / Centers for Disease Control and Prevention. https://data.cdc.gov/. Accessed 5 Apr 2018.

3. Dataset Compendium Overview /sgim.org. https://www.sgim.org/ communities/research/dataset-compendium. Accessed 5 Apr 2018

4. Musen MA, Bean CA, Cheung K-H, Dumontier M, Durante KA, Gevaert O, Gonzalez-Beltran A, Khatri P, Kleinstein SH, O'Connor MJ, Pouliot Y, Rocca-Serra P, Sansone S-A, Wiser JA, Team atC. The center for expanded data annotation and retrieval. J Am Med Inform Assoc. 2015;22(6):1148-52. https://doi.org/doi:10.1093/jamia/ocv048.
5. Gonçalves RS, O'Connor MJ, Martínez-Romero M, Egyedi AL, Willrett D, Graybeal J, Musen MA. The CEDAR Workbench: An Ontology-Assisted Environment for Authoring Metadata that Describe Scientific Experiments. In: The Semantic Web - ISWC 2017. Lecture Notes in Computer Science; 2017. p. 103-10. https://doi.org/10.1007/978-3-319-68204-4_10.

6. bioCADDIE | Biomedical and healthCAre Data Discovery and Indexing Ecosystem. https://biocaddie.org/. Accessed 3 Mar 2017.

7. Ohno-Machado L, Sansone S-A, Alter G, Fore I, Grethe J, Xu H, GonzalezBeltran A, Rocca-Serra P, Gururaj AE, Bell E, Soysal E, Zong N, Kim H-E. Finding useful data across multiple biomedical data repositories using DataMed. Nat Genet. 2017;49(6):816-9. https://doi.org/10.1038/ng.3864.

8. Berners-Lee T, Hendler J, Lassila O. The semantic web. Sci Am. 2001;284(5):28-37.

9. Dumontier M, Gray AJG, Marshall MS, Alexiev V, Ansell P, Bader G, Baran J, Bolleman JT, Callahan A, Cruz-Toledo J, Gaudet P, Gombocz EA, Gonzalez-Beltran AN, Groth P, Haendel M, Ito M, Jupp S, Juty N, Katayama T, Kobayashi N, Krishnaswami $K$, Laibe $C$, Le Novère $N$, Lin $S$, Malone J, Miller M, Mungall CJ, Rietveld L, Wimalaratne SM, Yamaguchi A. The health care and life sciences community profile for dataset descriptions. PeerJ. 2016;4. https://doi.org/10.7717/peerj.2331.

10. Miller E. An introduction to the resource description framework. Bull Am Soc Inf Sci Technol. 1998;25(1):15-9.

11. Prud'Hommeaux E, Seaborne A. SPARQL query language for RDF. W3C Recomm. 2008;15:.

12. Mendes PN, Jakob M, Bizer C. DBpedia: A Multilingual Cross-domain Knowledge Base,. In: LREC; 2012. p. 1813-7.

13. Larson SD, Martone ME. NeuroLex.org: an online framework for neuroscience knowledge. Front Neuroinformatics. 2013;7(18).

14. Wilkinson MD, Dumontier M, Aalbersberg IJ, Appleton G, Axton M, Baak A, Blomberg N, Boiten J-W, da Silva Santos LB, Bourne PE. The FAIR Guiding Principles for scientific data management and stewardship. Sci. 2016;3:

15. Krötzsch M, Vrandečić $D$, Völkel M. Semantic mediawiki. In: The Semantic Web-ISWC 2006; 2006. p. 935-42.

16. MediaWiki. https://www.mediawiki.org/wiki/MediaWiki. Accessed 3 Aug 2017.

17. Ciccarese P, Soiland-Reyes S, Belhajjame K, Gray AJ, Goble C, ClarkT. PAV ontology: provenance, authoring and versioning. J Biomed Semant. 2013;4(1):37. https://doi.org/10.1186/2041-1480-4-37.

18. Data Catalog Vocabulary (DCAT). https://www.w3.org/TR/vocab-dcat/. Accessed 3 Aug 2017.

19. CiTO, the Citation Typing Ontology. http://www.sparontologies.net/ ontologies/cito/source.html. Accessed 3 Aug 2017.

20. Shi J, Zheng M, Yao L, Ge Y. A Publication-based Popularity Index (PPI) for Healthcare Dataset Ranking. In: 2018 IEEE International Conference on Healthcare Informatics (ICHI); 2018. p. 247-54. https://doi.org/10.1109/ ICHI.2018.00035.

21. Steiner $C$, Elixhauser $A$, Schnaier J. The healthcare cost and utilization project: an overview. Eff Clin Pract: ECP. 2001;5(3):143-51.

22. MarketScan Research Data. https://marketscan.truvenhealth.com/ marketscanportal/. Accessed 3 Aug 2017.

23. Johnson AEW, Pollard TJ, Shen L, Lehman L-wH, Feng $M$, Ghassemi $M$, Moody B, Szolovits P, Celi LA, Mark RG. MIMIC-III, a freely accessible critical care database. Sci Data. 2016;3:160035. https://doi.org/10.1038/ sdata.2016.35.

24. Shi J, Zheng M, Yao L, Ge Y. DIR - A semantic information resource for healthcare datasets. In: 2017 IEEE International Conference on Bioinformatics and Biomedicine (BIBM); 2017. p. 805-10. https://doi.org/ 10.1109/BIBM.2017.8217758.

25. Health Informatics and Outcomes Research Academy | UNC Charlotte. https://hinora.uncc.edu/. Accessed 1 Apr 2017.

26. NHANES - National Health and Nutrition Examination Survey Homepage. https://www.cdc.gov/nchs/nhanes/index.htm. Accessed 1 Apr 2017.

27. SEER-Medicare Linked Database. https://healthcaredelivery.cancer.gov/ seermedicare/. Accessed 1 Apr 2017.

28. Add Health. http://www.cpc.unc.edu/projects/addhealth. Accessed 1 Apr 2017.

29. Minimum Data Set 3.0 Public Reports Overview. https://www.cms.gov/ Research-Statistics-Data-and-Systems/Computer-Data-an\%d-Systems/ Minimum-Data-Set-3-0-Public-Reports/index.html. Accessed 1 Apr 2017.

30. Clinical Practice Research Datalink - CPRD. https://www.cprd.com/home/. Accessed 1 Apr 2017.

31. THIN Database. https://www.ucl.ac.uk/pcph/research-groups-themes/ thin-pub/database. Accessed 1 Apr 2017. 
32. Premier Healthcare Database White Paper. https://learn.premierinc.com/ pharmacy-and-research/premier-healthcare-database-whitepaper. Accessed 1 Apr 2017.

33. Clinformatics Data Mart. http://www.optum.ca/life-sciences/ differentiate-products/marketing-analytics/clinformatics-data-mart.html. Accessed 1 Apr 2017.

34. Humedica NorthStar. https://www.optum.com/solutions/life-sciences/ explore-data/advanced-analytics/humedica-northstar.html. Accessed 1 Apr 2017.

35. Home - PMC - NCBI. https://www.ncbi.nlm.nih.gov/pmc/. Accessed 1 Apr 2017.

36. Shi J. Method Ontology. https://cci-hit.uncc.edu/dir/ontologies/ MethodOntology.owl. Accessed 3 Apr 2017.

37. Keet CM, Ławrynowicz A, d'Amato C, Kalousis A, Nguyen P, Palma R, Stevens R, Hilario M. The Data Mining OPtimization Ontology. Web Semant Sci Serv Agents World Wide Web. 2015;32:43-53. https://doi.org/ 10.1016/j.websem.2015.01.001.

38. Soysal E, Wang J, Jiang M, Wu Y, Pakhomov S, Liu H, Xu H. CLAMP - a toolkit for efficiently building customized clinical natural language processing pipelines. J Am Med Inform Assoc. https://doi.org/doi:10. 1093/jamia/ocx132

39. Smith AK, Ayanian JZ, Covinsky KE, Landon BE, McCarthy EP, Wee CC, Steinman MA. Conducting High-Value Secondary Dataset Analysis: An Introductory Guide and Resources. J Gen Intern Med. 2011;26(8):920-29. https://doi.org/10.1007/s11606-010-1621-5.

40. Quora - The Best Answer to Any Question. https://www.quora.com/. Accessed 3 Aug 2017.

41. Hot Questions - Stack Exchange. http://stackexchange.com/. Accessed 3 Aug 2017.

42. Speck R, Röder M, Oramas S, Espinosa-Anke L, Ngomo A-CN. Open Knowledge Extraction Challenge 2017. In: Semantic Web Challenges. Communications in Computer and Information Science. 2017. 35-48 https://doi.org/10.1007/978-3-319-69146-6_4.

43. Usbeck R, Ngomo A-CN, Haarmann B, Krithara A, Röder M, Napolitano G 7th Open Challenge on Question Answering over Linked Data (QALD-7). In: Semantic Web Challenges. Communications in Computer and Information Science; 2017. p. 59-69. https://doi.org/10.1007/978-3-31969146-6_6.

44. Lopez V, Uren V, Sabou M, Motta E. Is question answering fit for the semantic web?: a survey. Semant Web. 2011;2(2):125-55.

45. Jeon J, Croft WB, Lee JH. Finding similar questions in large question and answer archives. In: Proceedings of the 14th ACM International Conference on Information and Knowledge Management. 2005. 84-90.

46. Androutsopoulos I, Ritchie Gd, Thanisch P. Natural language interfaces to databases - an introduction. Nat Lang Eng. 1995;1 (01):29-81. https:// doi.org/10.1017/S135132490000005X.

47. Hirschman L, Gaizauskas R. Natural language question answering: the view from here. Nat Lang Eng. 2001;7(4):275-300.

48. Shekarpour S, Lukovnikov D, Kumar AJ, Endris K, Singh $\mathrm{K}$, Thakkar $\mathrm{H}_{\text {, }}$ Lange C. Question Answering on Linked Data: Challenges and Future Directions. arXiv:1601.03541 [Cs]; 2016. arXiv: 1601.03541.

Ready to submit your research? Choose BMC and benefit from:

- fast, convenient online submission

- thorough peer review by experienced researchers in your field

- rapid publication on acceptance

- support for research data, including large and complex data types

- gold Open Access which fosters wider collaboration and increased citations

- maximum visibility for your research: over $100 \mathrm{M}$ website views per year

At BMC, research is always in progress.

Learn more biomedcentral.com/submissions 